\title{
On the Interplay between Scheduling Interval and Beamwidth Selection for Low-Latency and Reliable V2V mmWave Communications
}

\author{
Cristina Perfecto*, Javier Del Ser ${ }^{* \dagger \ddagger}$ and Mehdi Bennis ${ }^{\S}$ \\ * University of the Basque Country (UPV/EHU), E-48013 Bilbao, Spain \\ Email: \{cristina.perfecto, javier.delser\}@ehu.eus \\ $\dagger$ TECNALIA, E-48160 Derio, Spain. Email: javier.delser@tecnalia.com \\ $\ddagger$ Basque Center for Applied Mathematics (BCAM), E-48009 Bilbao, Spain \\ $\S$ Centre for Wireless Communications, University of Oulu, FI-90014 Oulu, Finland \\ Email: bennis@ee.oulu.fi
}

\begin{abstract}
The interest in mmWave communications has risen sharply in the last years motivated by their widespread consideration as a technological solution capable of dealing with the stringent rate requirements currently demanded by wireless networks. This momentum gained by mmWave bands springs several technical challenges regarding the allocation of radio resources particularly complex in $\mathrm{V} 2 \mathrm{~V}$ communications, where reliability/latency constraints are extremely demanding, and links between vehicles are highly influenced by their mobility, beam misalignment and blockage between counterparts. In this context we analyze the interplay between the beamwidth assignment and the scheduling period when links between transmitters and receivers in $\mathrm{V} 2 \mathrm{~V}$ communications are established in a distributed fashion by means of Matching Theory. Extensive simulations performed for the aforementioned scheme and other alternatives from the literature reveal that, even in simplistic vehicular setups, the throughput performance and the latency/reliability trade-off is affected not only by the selected antenna beamwidths -and their suitability to the radio conditions imposed by the dynamics of the scenario under analysis- but also by a proper choice of the scheduling interval/beam realignment period. A poor choice in the latter for a given beamwidth being responsible of drop events increasing as much as $33 \%$ mainly due to beam misalignment.

Index Terms-V2V Communications, Millimeter-Wave, 5G, Matching Theory.
\end{abstract}

\section{INTRODUCTION}

In the last three years wireless communications deployed in the millimeter-wave (mmWave) have rapidly become a key enabler to satisfy the stringent requirements of 5G networks [1], [2]. At the same time, the realization of the critical part that seamless connectivity will play to achieve higher automation levels [3] has turned the attention of the automotive sector towards the use of mmWave for vehicular communications. The integration of assorted wireless technologies in vehicles for applications related to safety and leisure (infotainment) together with increased sensor deployment [4] are foreseen to require very high transmission rates exceeding both the admitted limit of $27 \mathrm{Mbps}$ of DSRC [5] or current 4G cellular communications rates. The envisaged extensive use of advanced radar technologies [6] such as LIDAR to produce high-resolution maps require even more demanding data rates that further motivate the adoption of mmWave for vehicleto-everything (V2X) communications. Recent works such as [6], [7] have highlighted this spectrum band to support highbandwidth automotive sensing or beamforming in vehicleto-infrastructure (V2I) communications [8]. However, to the best of our knowledge the literature on the performance of mmWave V2V communications is so far limited to [9], which explored the impact of directionality and blockage on the signal to interference plus noise ratio (SINR) with a static vehicle association approach. Nonetheless this work employed naïve traffic generation approaches in the two scenarios that it proposed, and did not cover aspects of paramount importance in the automotive sector such as the delay and reliability performance associated to those data arrival processes.

The need to resort to dynamic radio resource management (RRM) policies adapting to both channel and queuig state information (CSI/QSI) to optimize delay performance while guaranteeing extremely high reliability metrics motivated our previous recent work in [10]. Therein we proposed and evaluated a cross-layer and distributed RRM comprised of a matching theory based V2V link selection framework that was augmented either with a beamwidth optimization stage or with the use of fixed beamwidths. This present work builds upon the latter to explore how the enforcement period of the RRM becomes further critical due to the interplay between how often links are reorganized in the network -considering that signalling overheads incurred by instantaneous reporting of CSI/QSI are to be minimized-, and the likelihood of triggering misalignment events if the steering of the antennas in $\mathrm{V} 2 \mathrm{~V}$ links is not performed often enough in the context of the dynamic topology entailed by the movement of vehicles.

The rest of the paper is organized as follows: Section II delves into the system model and problem formulation. For the sake of completeness, Section III and subsections therein provide a detailed explanation on the proposed resource allocation procedure. Section IV presents and discusses simulation results. Finally, Section V concludes the paper outlining some related lines of future research. 


\section{System Model And Problem Statement}

We consider a multiple lane highway section where vehicles incorporate vehicular user equipments (vUEs) acting as either transmitters (vTx) or receivers (vRx) communicating through $\mathrm{V} 2 \mathrm{~V}$ links on the mmWave frequency band. Time Division Duplexing (TDD), a co-channel deployment with bandwidth $B$, uniform transmit power and half-duplex modes are also hereafter assumed. Let $\mathcal{I} \triangleq\{1, \ldots, I\}, \mathcal{J} \triangleq\{1, \ldots, J\}$ and $\mathcal{L} \triangleq\{1, \ldots, L\}$ respectively denote the sets of vTx, vRx and links in the system, with $\mathcal{I} \cap \mathcal{J}=\emptyset,|\mathcal{L}| \leq \min \{|\mathcal{I}|,|\mathcal{J}|\}$ and $|\cdot|$ denoting set cardinality. Due to the complexity of instantaneous uncoordinated RRM policies, time-slotted communications with two time scales will be hereafter considered:

- Data transmission slots, to denote the interval $\left[t, t+T_{t}\right)$ with $T_{t}$ as the duration of the transmission period.

- Scheduling slots, to refer to the interval $\left[t, t+T_{s}\right)$ with $T_{s}$ representing the duration of the network-wide enforced control actions.

Each scheduling slot will comprise $N$ transmission slots (i.e. $T_{s}=N T_{t}$ ) such that scheduling is performed at $\mathcal{T}_{s} \triangleq$ $\left\{t_{s} \in \mathbb{N}: t_{s} \bmod N=0\right\}$ and data transmission is held at $\mathcal{T}_{t} \triangleq \mathbb{N} \cup\{0\}$.

\section{A. Channel and Sectored Antenna Models}

The log-distance pathloss model proposed in [11] and extended in [9] for an unlimited number of blockers ${ }^{1}$ obtruding the $\mathrm{V} 2 \mathrm{~V}$ link is adopted. The channel gain $g_{i, j}^{c}$ of link $\ell_{i, j}$ between $\operatorname{vTx} i$ and $\operatorname{vRx} j$ is given by

$$
g_{i, j}^{c}=10 \alpha_{i, j} \log _{10}\left(\mathrm{~d}_{i, j}\right)+\beta_{i, j}+15 \mathrm{~d}_{i, j} / 1000,
$$

with the third term representing the atmospheric attenuation at $60 \mathrm{GHz}$, and the values for $\alpha_{i, j}$ and $\beta_{i, j}$ specified by the number of blockers in the link $\ell_{i, j}$.

Since we deal with a dynamic scenario the channel gain may vary as a result of the relative movement of the vehicles, which yields $g_{i, j}^{c}(t)$. At the end of a transmission slot $t \in \mathcal{T}_{t}$ the aggregate global $\mathrm{CSI}^{2}$ for the set of $|\mathcal{J}|$ receivers will be given by $\mathbf{H}_{\mathcal{J}}(t)=\left\{H_{j}(t): \forall j \in \mathcal{J}\right\}$, with $H_{j}(t)=g_{i, j}^{c}(t)$ if link $\ell_{i, j}$ exists.

Directional antenna patterns in vehicles can be approximated by a two-dimensional sectored antenna model that captures the most relevant features of the radiation pattern, i.e. the boresight direction, the half-power beamwidth and frontto-back ratio for directivity gains in the mainlobe and sidelobe as shown in Fig. 1. Transmission and reception directivity gains $g_{i, j}^{\wp}(t)\left(\wp \in\left\{t_{x}, r_{x}\right\}\right)$ of vehicles in link $\ell_{i, j}$ during a transmission slot $t \in \mathcal{T}_{t}$ are given by

$g_{i, j}^{\wp}(t)= \begin{cases}G\left(\varphi_{i, j}^{\wp}\right)=\frac{2 \pi-\left(2 \pi-\varphi_{i, j}^{\wp}(t)\right) g_{\varangle}}{\varphi_{i, j}^{\wp}(t)}, & \text { if }\left|\theta_{i, j}^{\wp}(t)\right| \leq \varphi_{i, j}^{\wp} / 2, \\ g_{\varangle}, & \text { otherwise, }\end{cases}$

\footnotetext{
${ }^{1}$ The effect of blockage is here considered to be mitigated by using antennas located at bumper-level and having the gap under vehicles acting as waveguard which effectively allows to establish links among non-adjacent vehicles.

${ }^{2}$ Instantaneous reporting of CSI and QSI related side effects (e.g. increased signaling overhead) will be avoided by enforcing a long-term RRM strategy that includes, among others, learning techniques.
}

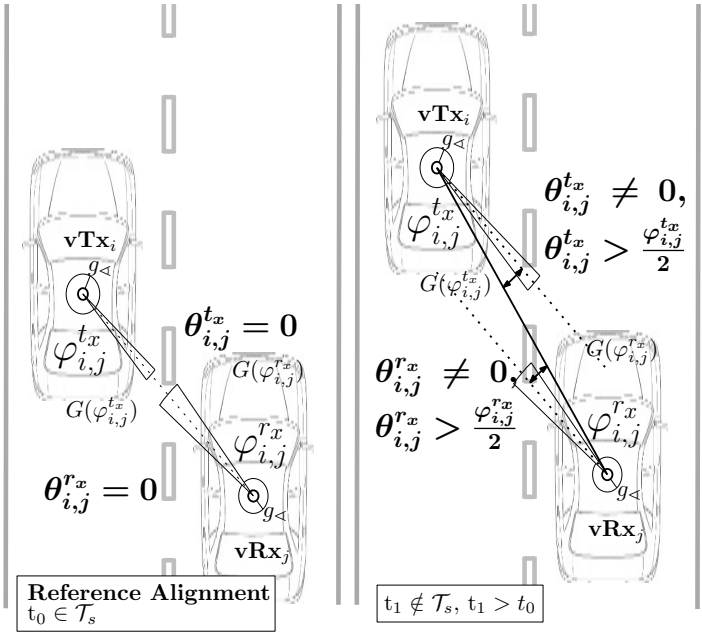

Fig. 1. Elements of the sectored antenna model and the effect of the misalignment between transmitter and receiver boresight directions on the vTx and VRx antenna gains.

where $\theta_{i, j}^{\wp}(t)$ represents the alignment error between the antenna steering directions and their corresponding boresight directions of $\operatorname{vTx} i$ and $\operatorname{vRx} j ; \varphi_{i, j}^{\wp}(t)$ is the half-power beamwidth of link $\ell_{i, j}$ at transmission $\left(\wp=t_{x}\right)$ and reception $\left(\wp=r_{x}\right)$ sides established for the scheduling period at hand; and $0 \leq g_{\varangle} \ll 1$ is the non-negligible sidelobe power.

\section{B. Alignment Delay and Transmission Rate}

A two-staged beam alignment mechanism, simplified from the beam codebook-based method introduced by [12], is considered. In this procedure a sequence of pilot transmissions and a trial-and-error approach eventually produces the best steering for the beams at both ends of the V2V link. Without loss of generality, we consider here that for any two vehicles in a V2V link before the beam-level alignment phase itself, either a sector-level alignment has been previously performed or that a coarse location of neighboring vehicles has been learned effectively reducing the beam search. By applying a continuous approximation [13], the alignment time penalty $\tau_{i, j}(t)$ can be expressed as

$$
\tau_{i, j}(t) \triangleq \tau_{i, j}\left(\varphi_{i, j}^{t_{x}}(t), \varphi_{i, j}^{r_{x}}(t)\right)=\frac{\psi_{i}^{t_{x}} \psi_{j}^{r_{x}}}{\varphi_{i, j}^{t_{x}}(t) \varphi_{i, j}^{r_{x}}(t)} T_{p}
$$

where $\psi_{i}^{t_{x}}$ and $\psi_{j}^{r_{x}}$ denote the sector-level beamwidths of vTx $i$ and $\operatorname{vRx} j$, and $T_{p}$ denotes the pilot transmission duration. Under these assumptions the maximum achievable data rate $r_{i, j}(t)$ between vTx $i$ and $\operatorname{vRx} j$ will depend on whether beam alignment is performed at time slot $t$ with its corresponding induced delay and on the measured SINR at vRx $j$, including the interference of other incumbent vTxs $\mathcal{Z}$ on vRx $j$. The rate for a time slot $t$ of duration $T_{t}$ will be then

$$
r_{i, j}(t)=\left(1-\frac{\tau_{i, j}(t)}{T_{t}}\right) B \log _{2}\left(1+\operatorname{SINR}_{j}(t)\right),
$$


where the SINR term over the slot $t$ for $Z=|\mathcal{Z}|$ simultaneously transmitting vTxs is given by

$$
\operatorname{SINR}_{j}(t)=\frac{p_{i} g_{i, j}^{t_{x}}(t) g_{i, j}^{c}(t) g_{i, j}^{r_{x}}(t)}{\sum_{\substack{z \in \mathcal{Z} \subseteq \mathcal{I} \\ z \neq i}} p_{z} g_{z, j}^{t_{x}}(t) g_{z, j}^{c}(t) g_{z, j}^{r_{x}}(t)+N_{0} B},
$$

with $p_{i}$ as the transmission power of reference vTx $i$ (correspondingly, $p_{z}$ the transmission power of interfering vTx $z$ ); $g_{i, j}^{c}(t)$ the channel gain in the link $\ell_{i, j} ; g_{i, j}^{t_{x}}(t)$ and $g_{i, j}^{r_{x}}(t)$ denoting the antenna gains of vehicles at both ends of link $\ell_{i, j}$; and $N_{0}$ the Gaussian background noise power density in $\mathrm{dBm} / \mathrm{Hz}$. Finally, it is also straightforward to note that the rate $r_{i, j}(t)$ increases when no alignment is performed during the time slot $t$, as per Expression (4) with $\tau_{i, j}(t)=0$. From the above formulae it can be noted the well-known alignment delay versus throughput trade-off [13], under which narrower beamwidths involve exploring more steering possibilities and consequently, longer training overheads that reduce the effective transmission rate of the link.

\section{Queues and Delay Modeling}

As mentioned in the introduction, this paper focuses on adaptive RRM policies that leverage CSI/QSI information learned prior to the scheduling itself. To this end, a model for the traffic and queue dynamics is required: this will be accomplished by assuming that each vTx maintains a queue for data coming from upper layers of the protocol stack, e.g. data from sensors. Let $Q_{i}(t)$ be the queue length (in number of packets) of vTx $i$ at the beginning of time slot $t$, and let $\mathbf{A}_{\mathcal{I}}(t)=\left(A_{1}(t), \ldots, A_{I}(t)\right)$ denote the random packet arrival vector (in number of packets) to the set $\mathcal{I}$ of vTxs at the end of time slot $t \in \mathcal{T}_{t}$. Every entry $A_{i}(t)$ in $\mathbf{A}_{\mathcal{I}}(t)$ is assumed to be i.i.d. over different time slots as a result of random, mutually independent message arrival processes following a Poisson distribution with mean $\lambda \forall i \in\{1, \ldots, I\}$. We further consider a fixed packet size $P_{s}$. Then, if $R_{i}(t)$ packets are successfully transmitted on link $\ell_{i, j}$ during time slot $t \in \mathcal{T}_{t}$, the queue dynamics for vTx $i$ are given by

$$
Q_{i}(t+1)=\min \left\{\left(Q_{i}(t)-R_{i}(t)\right)^{+}+A_{i}(t), Q_{\max }\right\},
$$

where $x^{+} \triangleq \max \{x, 0\}$ and $Q_{\max }$ is the maximum buffer size (in number of packets) of the queue. We let $\mathbf{Q}_{\mathcal{I}}(t)=\left\{Q_{i}(t)\right.$ : $\forall i \in \mathcal{I}\}$ denote the aggregate global QSI vector for the set $\mathcal{I}$ of vTxs at the beginning of time slot $t \in \mathcal{T}_{t}$. So that, we define the global system state at time slot $t \in \mathcal{T}_{t}$ as $\mathcal{X}(t) \triangleq$ $\left(\mathbf{H}_{\mathcal{J}}(t), \mathbf{Q}_{\mathcal{I}}(t)\right) \in \Upsilon$ with $\Upsilon \triangleq \mathcal{X}^{1} \cup \mathcal{X}^{2} \ldots$ representing the global system state space such that $|\Upsilon|=\left(Q_{\max }+1\right)^{L}$.

Upon its arrival to a certain queue, a packet will be either delivered or dropped within $D_{\max }^{\lambda} \mathrm{ms}$ after entering the queue:

- If link $\ell_{i, j}$ is active and channel conditions in the link allow, packet $P_{i}^{p}$ (with $p \in\left\{1, \ldots, A_{i}(t)\right\}$ ) will be transmitted with a delay $d_{i, j}^{p} \leq D_{\max }^{\lambda}$ given by

$$
d_{i, j}^{p}=t_{i}^{p, s e r v}-t_{i}^{p, a r r},
$$

with $t_{i}^{p, a r r}, t_{i}^{p, s e r v}$ respectively standing for the arrival time of packet $P_{i}^{p}$ at the queue of vTx $i$ and the time when the last of the bits of $P_{i}^{p}$ is transmitted to vRx $j$, i.e, $d_{i, j}^{p}$ is a measure of the joint queue waiting and queue service time. If we denote $\mathcal{A}_{i}^{\checkmark}(t)$ as the subset of packets successfully sent towards $\mathrm{vRx} j$ at time $t \in \mathcal{T}_{t}$, then the average delay per packet $\bar{D}_{i, j}(t)$ during transmission slot $t \in \mathcal{T}_{t}$ can be computed by averaging the delays $d_{i, j}^{p}$ of each packet successfully delivered over this link for the slot at hand, as

$$
\bar{D}_{i, j}(t)=\frac{\sum_{p \in \mathcal{A}_{i}^{\curlyvee}(t)} d_{i, j}^{p}}{\left|\mathcal{A}_{i}^{\curlyvee}(t)\right|} .
$$

The average delay per delivered packet over the scheduling period $t_{s} \in \mathcal{T}_{s}$ will be thus given by

$$
\bar{D}_{i, j}^{s c h}\left(t_{s}\right)=\frac{\sum_{t=t_{s}-N+1}^{t_{s}} \bar{D}_{i, j}(t)}{N} .
$$

- If link $\ell_{i, j}$ is active but channel conditions in the link do not suffice for delivering the entire packet to receiver $\mathrm{vRx} j$ within $D_{\max }^{\lambda}$, the packet will be dropped from the queue. The rationale behind the adoption of this hard operational directive is that we target ultrareliable low-latency communications by which newer traffic should be prioritized so as to ensure minimumdelay communications. Each time a packet is dropped a penalty will be incurred and computed in the form of a reliability loss. Specifically, the set of dropped packets in a transmission slot $t \in \mathcal{T}_{t}$ will be denoted as $\mathcal{A}_{i}^{\times}(t)$, such that $\mathcal{A}_{i}^{\times}(t) \cup \mathcal{A}_{i}^{\vee}(t)=\mathcal{A}_{i}(t)$ and $\mathcal{A}_{i}^{\times}(t) \cap \mathcal{A}_{i}^{\checkmark}(t)=\emptyset$.

\section{Problem Statement}

A RRM policy can be mathematically casted by first defining $\boldsymbol{\Phi}\left(t_{s}\right) \triangleq\left\{\phi_{i, j}\left(t_{s}\right): i \in \mathcal{I}\left(t_{s}\right), j \in \mathcal{J}\left(t_{s}\right)\right\}$ as the set of all possible vTx/vRx pairs in the system within a given scheduling slot $t_{s} \in \mathcal{T}_{s}$. Note here that $\mathcal{I}\left(t_{s}\right)$ (corr. $\mathcal{J}\left(t_{s}\right)$ ) denotes the subset of vTx and vRx present on the road scenario at scheduling time $t_{s}$. By slightly expanding prior notation $\mathcal{I}_{j}\left(t_{s}\right) \subseteq \mathcal{I}\left(t_{s}\right)$ and $\mathcal{J}_{i}\left(t_{s}\right) \subseteq \mathcal{J}\left(t_{s}\right)$ respectively denote the subset of feasible vTxs for vRx $j$ and the feasible vRxs for vTx $i$, where feasibility is imposed by a circular coverage constraint of radius $R_{c}$ (in meters). In this set $\phi_{i, j}\left(t_{s}\right)$ will represent the association variable so that for the vehicle pair composed by vTx $i$ and $\mathrm{vRx} j$,

$$
\phi_{i, j}\left(t_{s}\right)= \begin{cases}1 & \text { if link } \ell_{i, j} \text { is set } \forall t \in\left(t_{s}-N, t_{s}\right], \\ 0 & \text { otherwise. }\end{cases}
$$

As was exemplified in Fig. 1, the likeliness of misalignment and its eventual impact on desired links due to a non-continuous steering/beamtracking mechanism may vary depending on several factors such as the relative speed of the vehicles involved in the link, the width of the mainlobes of transmitter and receiver antennas and/or the length of the scheduling interval. Moreover, the beamwidths will impel whether signals from undesired V2V links arrive into the sidelobes or the mainlobe of vRxs, which may severely degrade the measured SINR levels. Without loss of generality, for $\varphi_{i, j}^{t_{x}}(t)=\varphi_{i, j}^{r_{x}}(t)=\varphi \forall i, j$ and $\forall t$, the effective instantaneous 
rate $r_{i, j}(t)$ of link $\ell_{i, j}$ will be given by Expressions (4) and (5) with $\mathcal{Z}=\mathcal{I}\left(t_{s}\right)$ and relative interferences and gains between pairs given by the prevailing matching policy $\boldsymbol{\Phi}\left(t_{s}\right)$, i.e. for if $t \in\left[t_{s}, t_{s}+T_{t}\right)$,

$$
r_{i, j}(t)=\left(1-\frac{\tau_{i, j}(t)}{T_{t}}\right) B \log _{2}\left(1+\operatorname{SINR}_{j}(t)\right),
$$

while for $t \in\left[t_{s}+T_{t}, t_{s}+N T_{t}\right)$,

$$
r_{i, j}(t)=B \log _{2}\left(1+\operatorname{SINR}_{j}(t)\right) .
$$

Depending on this rate and the mean packet inter-arrival time $\lambda$, a fraction of the packets generated at vTx $i$ will be transmitted towards $\mathrm{vRx} j$, producing different delays and packet drop statistics over the given scheduling slot. On one hand, the average delay per packet $\bar{D}_{i, j}(t)$ will increase as $r_{i, j}(t)$ is lower, whereas the packet drop rate, defined as

$$
\Gamma_{i}^{\times}(t) \triangleq \frac{\left|\mathcal{A}_{i}^{\times}(t)\right|}{\left|\mathcal{A}_{i}^{\times}(t)\right|+\left|\mathcal{A}_{i}^{\checkmark}(t)\right|}=\frac{\left|\mathcal{A}_{i}^{\times}(t)\right|}{A_{i}(t)},
$$

will increase whenever the average packet arrival rate $1 / \lambda$ is high enough and/or $r_{i, j}(t)$ is low to cause massive packet drops. In both cases, it should be clear that a delay-sensitive RRM policy should take into account not only the finite delay statistics of those packets successfully transmitted towards their destinations (for which queue dynamics are set to prioritize new incoming traffic), but also the interplay between delay and dropped packets enforced by the queuing policy.

The problem tackled in this work can be hence formulated as the selection of the RRM policy $\boldsymbol{\Phi}\left(t_{s}\right)$ for $t_{s} \in \mathcal{T}_{s}$ such that

$$
\begin{aligned}
& \underset{\boldsymbol{\Phi}\left(t_{s}\right)}{\operatorname{Minimize}} \sum_{i \in \mathcal{I}\left(t_{s}\right)} \sum_{j \in \mathcal{J}\left(t_{s}\right)} \bar{D}_{i, j}^{s c h}\left(t_{s}\right) \phi_{i, j}\left(t_{s}\right), \\
& \text { subject to: } \bar{Q}_{i, j}(t)<\infty \forall t \in\left(t_{s}-N, t_{s}\right], \\
& \sum_{j \in \mathcal{J}\left(t_{s}\right)} \phi_{i, j}\left(t_{s}\right)=1, \forall i \in \mathcal{I}\left(t_{s}\right), \\
& \sum_{i \in \mathcal{I}\left(t_{s}\right)} \phi_{i, j}\left(t_{s}\right)=1, \forall j \in \mathcal{J}\left(t_{s}\right), \\
& \phi_{i, j}\left(t_{s}\right) \in\{0,1\}, \forall i, j \in \mathcal{I}\left(t_{s}\right) \times \mathcal{J}\left(t_{s}\right),
\end{aligned}
$$

where inequality (14b) indicates that no queue should overflow during the scheduling period at hand, and Expressions (14c) through (14e) denote that vehicles are paired one-to-one.

\section{Distributed Association Via Matching Theory}

The optimization problem formulated in the previous section is difficult to tackle analytically. Furthermore, in vehicular scenarios the design target should be steered towards lowcomplexity distributed solutions so as to avoid traffic overheads that could eventually compromise the end-to-end delay statistics of the deployed links. Based on this rationale in [10] we explored the framework of Matching Theory [14] to undertake the distributed optimization of $\boldsymbol{\Phi}\left(t_{s}\right)$. It is important to remark at this point that the ultimate purpose of this research study is to assess the performance figures of different
RRM strategies, with an emphasis on reliability/delay metrics and always considering the RRM enforcing time - namely, the length of the scheduling interval $T_{s}-$ as the driver of our analysis. In this regard, although several algorithmic alternatives from the literature will be included in the simulation benchmark later discussed in the paper, conclusions will gravitate not only on the relative performance gains among distributed association schemes, but also on the dependence of the obtained metrics with $T_{s}$ and its consistence over such pairing methods.

\section{A. Matching Game: Definitions}

Several definitions must be first done to properly grasp the fundamentals of this mathematical framework and put them in context of this manuscript:

Definition 1: A matching game is defined by two sets of players $\left(\mathcal{I}_{j}(t), \mathcal{J}_{i}(t)\right)$ and two preference relations $\succ_{i}, \succ_{j}$, allowing each player $i \in \mathcal{I}_{j}(t), j \in \mathcal{J}_{i}(t)$ to accordingly rank the players in the opposite set.

Definition 2: A matching game is a matching function $\boldsymbol{\Phi}(t)=\left\{\phi_{i, j}(t)\right\}$ that bilaterally assigns players $\phi_{i}(t) \triangleq\{j \in$ $\left.\mathcal{J}_{i}(t): \phi_{i, j}(t)=1\right\}$ and $\phi_{j}(t) \triangleq\left\{i \in \mathcal{I}_{j}(t): \phi_{i, j}(t)=1\right\}$ such that $\left|\phi_{j}(t)\right|=q_{j}$ and $\left|\phi_{i}(t)\right|=q_{i}$. Here $q_{i}$ and $q_{j}$ stand for the quota of the player which, for a one-to-one matching, $q_{i}=q_{j}=1$.

Definition 3: A preference $\succ$ is a complete, reflexive and transitive binary relation between the players in $\mathcal{I}_{j}(t)$ and $\mathcal{J}_{i}(t)$. Therefore for any vTx $i$ a preference relation $\succ_{i}$ is defined over the set of vRx $\mathcal{J}_{i}(t)$ such that for any two vRx $(m, n) \in \mathcal{J}_{i}(t) \times \mathcal{J}_{i}(t)$ with $m \neq n$, and two matchings $\boldsymbol{\Phi}(t)$ and $\boldsymbol{\Phi}^{\prime}(t)$ so that $\phi_{i}(t)=m$ and $\phi_{i}^{\prime}(t)=n$ :

$$
(m, \boldsymbol{\Phi}(t)) \succ_{i}\left(n, \boldsymbol{\Phi}^{\prime}(t)\right) \Leftrightarrow U_{v T x}^{i, m}(t)>U_{v T x}^{i, n}(t) .
$$

And that, similarly, for any $\mathrm{vRx} j$ a preference relation $\succ_{j}$ is defined over the set of vTx $\mathcal{I}_{j}(t)$ such that for any two vTx $(k, l) \in \mathcal{I}_{j}(t) \times \mathcal{I}_{j}(t)$ with $k \neq l$, and two matchings $\boldsymbol{\Phi}(t)$ and $\boldsymbol{\Phi}^{\prime}(t)$ so that $\phi_{j}(t)=k$ and $\phi_{j}^{\prime}(t)=l$ :

$$
(k, \boldsymbol{\Phi}(t)) \succ_{j}\left(l, \boldsymbol{\Phi}^{\prime}(t)\right) \Leftrightarrow U_{v R x}^{j, k}(t)>U_{v R x}^{j, l}(t),
$$

where $U_{v T x}^{i, m}(t)$ and $U_{v R x}^{k, j}(t)$ denote the utility of $\mathrm{vRx} m$ for vTx $i$ and the utility of vTx $k$ for $\mathrm{vRx} j$, correspondingly.

Definition 4: A matching is not stable if for a given match $\phi_{i}(t)=j$ and $\phi_{j}(t)=i$, a blocking pair $\left(i^{\prime}, j^{\prime}\right)$ such that $i, i^{\prime} \in \mathcal{I}_{j}(t)$ and $j, j^{\prime} \in \mathcal{J}_{i}(t)$ satisfying $\phi_{i}(t) \neq j^{\prime}, \phi_{j}(t) \neq i^{\prime}$ and $j^{\prime} \succ_{i} j, i^{\prime} \succ_{j} i$ exists. That is, if for a given match two players prefer to be matched to each other over their current matched partners. A matching is considered to be pairwise stable if no such blocking pair exists.

Gale-Shapley's Deferred Acceptance (DA) algorithm [15] can be applied to solve one-to-one canonical matchings ${ }^{3}$. In essence it relies on an iterative process to find a pairwise stable mapping from the elements of the set of vTxs in the system to the elements of the set of feasible vRxs at every scheduling

\footnotetext{
${ }^{3}$ A Matching game is said to be canonical if the preferences of players are not influenced by any other player's decisions.
} 
period. The process sorts the preference list that each vehicle on either sides compiles over the vehicles from the other set. DA does not require a centralized controller as the players involved do not need to observe the actions or preferences of other players. Unfortunately, DA is unsuitable if the game is subject to externalities, i.e. interdependencies between the players' preferences.

The baseline for the formulation of utilities at both ends leading to the $\mathrm{V} 2 \mathrm{~V}$ link allocation will be the $\alpha$-fair utility function [16], expressed for $\alpha \geq 0$ and $\wp \in\{v T x, v R x\}$, as

$$
U_{\wp}\left(r_{\wp}(t)\right)=\omega_{x} \frac{r_{\wp}(t)^{1-\alpha_{\wp}}}{1-\alpha_{\wp}},
$$

which if $\alpha=2$ provides weighted minimum proportional delay fairness, and whose term $\omega_{\wp}$ allows to bring problemspecific knowledge into the utilities. We define the weighted $\alpha$-fair utility function for vTx $i \in \mathcal{I}\left(t_{s}\right)$ over vRxs $\mathcal{J}_{i}(t)$ as

$$
U_{v T x}^{i, j}\left(t_{s}\right) \triangleq-\frac{\omega_{v T x}^{i, j}\left(t_{s}\right)}{r_{i, j}\left(t_{s}, \mathbf{\Phi}\left(t_{s}\right)\right)},
$$

where, for notational simplicity, we will use $U_{v T x}^{i, j}\left(t_{s}\right)$ instead of $U_{v T x}^{i, j}\left(t_{s}, \mathbf{\Phi}\left(t_{s}\right)\right)$ leaving implicit the dependence of the utility on $\boldsymbol{\Phi}\left(t_{s}\right)$. Similarly, the utility of $\mathrm{vRx} j \in \mathcal{J}\left(t_{s}\right)$ over $I_{j}\left(t_{s}\right)$ vTxs for a given matching $\mathbf{\Phi}\left(t_{s}\right)$ will be

$$
U_{v R x}^{j, i}\left(t_{s}\right)=-\frac{\omega_{v R x}^{j, i}\left(t_{s}\right)}{r_{i, j}\left(t_{s}, \boldsymbol{\Phi}\left(t_{s}\right)\right)}
$$

so that the system welfare $\mathbf{S}\left(t_{s}, \mathbf{\Phi}\left(t_{s}\right)\right)$ is

$$
\mathbf{S}\left(t_{s}, \boldsymbol{\Phi}\left(t_{s}\right)\right) \triangleq \sum_{\mathcal{I}\left(t_{s}\right)} \sum_{\mathcal{J}_{i}\left(t_{s}\right)} \phi_{i, j}\left(t_{s}\right)\left(U_{v T x}^{i, j}\left(t_{s}\right)+U_{v R x}^{j, i}\left(t_{s}\right)\right) .
$$

Finally weights $\omega_{v T x}^{i, j}\left(t_{s}\right)$ and $\omega_{v R x}^{j, i}\left(t_{s}\right)$ are defined to reflect the traffic influx rate of the queuing system defined as the packet size $P_{s}$ times the average traffic arrival rate $1 / \lambda$. The latter formulation bring to light the link between the system welfare in (20) and the fitness function in (14): utility functions reflect the load of a given $\mathrm{V} 2 \mathrm{~V}$ link in terms of the number of transmission slots needed to serve $P_{s}$ bits with rate $r_{i, j}\left(t_{s}\right)$.

In practice, the need for information exchanges about the current matching state at an instantaneous scale would contradict our distributed approach to the problem. Moreover, the formulation of (18) and (19) reflects that the rate on a link $\ell_{i, j}$ will not only depend on the currently matched vTx but also on whom the rest of the vTxs are matched to, which unveils the existence of externalities. These externalities in our system are the result of the directionality of mmWave links and the variability of the levels of received interference built upon the beam steering. With the twofold aim of reducing the exchange of instantaneous CSI and QSI levels an estimate of $r_{i, j}\left(t_{s}, \mathbf{\Phi}\left(t_{s}\right)\right)$ all along the scheduling interval is carried out by using a link exploration and learning procedure over a mmWave control channel deployed in parallel to the main communication beam. Such a learning algorithm may hinge on diverse technical approaches, such as the sampling and averaging approach proposed in [10]. In any case the outcome
TABLE I

SIMULATION PARAMETERS

\begin{tabular}{|l|l|}
\hline Parameter & Value \\
\hline Simulation time & $30000 \mathrm{~ms}$ \\
\hline Transmission slot duration $T_{t}$ & $2 \mathrm{~ms}$ \\
\hline Scheduling slot duration $T_{s}$ & {$[50,100,200,500] \mathrm{ms}$} \\
\hline$[$ LOW/ULTRA] Vehicle Densities & {$[70 / 180]$ vehicles/km } \\
\hline Packet size $\mathrm{P}_{s}$ & {$[3200,2097144]^{4} \mathrm{bits}$} \\
\hline Lane Speed & {$[140,130,125,110,90,70] \mathrm{km} / \mathrm{h}$} \\
\hline Car to Truck ratio & $80 \%($ cars $), 20 \%($ trucks $)$ \\
\hline vTx/vRx probability & $50 \%(\mathrm{vTx}), 50 \%(\mathrm{vRx})$ \\
\hline Coverage radius $R_{c}$ & $100 \mathrm{~m}$ \\
\hline Peak transmit/slot time $\left(T_{p} / T_{s}\right)$ & 0.01 \\
\hline Transmit/receive beamwidth $\varphi$ & $5^{\circ}, 10^{\circ}, 45^{\circ}$ \\
\hline Sector-level beamwidth $\left(\psi_{i}^{t}, \psi_{i}^{r}\right)$ & $45^{\circ}$ \\
\hline Carrier frequency & $60 \mathrm{Ghz}$ \\
\hline Bandwidth $\left(\mathcal{B}_{m m w}\right)$ & $2.16 \mathrm{GHz}$ \\
\hline Noise Power Spectral density $\left(N_{0}\right)$ & $-174 \mathrm{dBm} / \mathrm{Hz}$ \\
\hline vTx transmit power $\left(p_{i}\right)$ & $15 \mathrm{dBm}$ \\
\hline
\end{tabular}

of the learning procedure provides values for $\bar{r}_{i, j}^{e s t}\left(t_{s}\right)$ to replace $r_{i, j}\left(t_{s}, \mathbf{\Phi}\left(t_{s}\right)\right)$ in (18) and (19).

Now that externalities have been removed from the rates of the system, the final utilities over which the matching game will be played are

$$
\begin{gathered}
U_{v T x}^{i, j}\left(t_{s}\right) \triangleq-\frac{\omega_{v T x}^{i, j}\left(t_{s}\right)}{\bar{r}_{i, j}^{e s t}\left(t_{s}\right)}=-\frac{P_{s} / \lambda}{\bar{r}_{i, j}^{e s t}\left(t_{s}\right)}, \\
U_{v R x}^{j, i}\left(t_{s}\right)=-\frac{\omega_{v R x}^{j, i}\left(t_{s}\right)}{\bar{r}_{i, j}^{e s t}\left(t_{s}\right)}=-\frac{P_{s} / \lambda}{\bar{r}_{i, j}^{e s t}\left(t_{s}\right)},
\end{gathered}
$$

As a result, the $\mathrm{V} 2 \mathrm{~V}$ mmWave link allocation problem can be cast as a one-to-one canonical matching game and solved in a distributed manner by using the aforementioned DA algorithm.

\section{Simulation Setup AND Results}

To shed light on how the scheduling period $T_{s}$ impacts the performance metrics of the overall vehicular scenario, a comprehensive set of computer simulations have been carried out over a 500 meter-length highway segment with 63 meter-wide lanes. Vehicles are either cars or trucks, with the former drawn uniformly at random from a set of 5 different models, each characterized by varying lengths and widths. Upon their entrance to the highway vehicles will be declared as transmitters (vTx) or receivers (vRx) with equal probability disregarding the role of vehicles that leave the system. To assess the impact of queue dynamics under different configurations several packet arrival rates and packet sizes are considered in two different traffic densities scenarios hereafter referred to as LOW and ULTRA vehicle densities. Transmit and receive beamwidths $\varphi_{i, j}^{t_{x}}(t)$ and $\varphi_{i, j}^{r_{x}}(t)$ of the mmWave channels are kept constant over $t$ and equal to $\varphi$ for every link.

\footnotetext{
${ }^{4}$ Packet sizes of $P_{s}=3200$ and $P_{s}=2097144$ bits are in line with the specifications for the DSRC safety messages length and the 802.11ad maximum payload, respectively.
} 


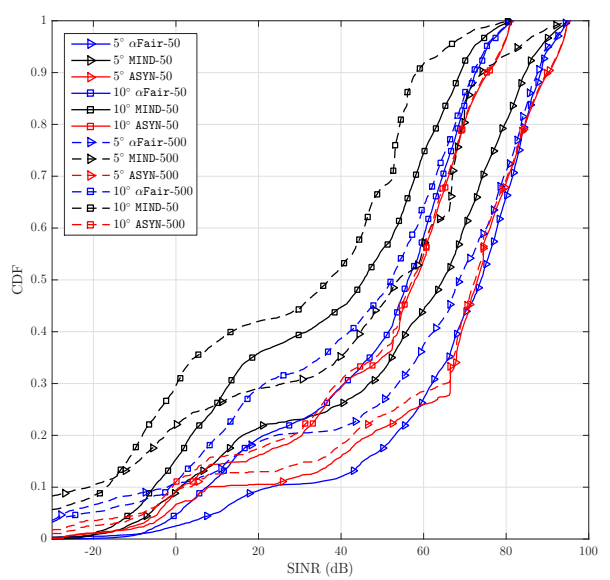

(a) SINR for $T_{s}=50 / 500 \mathrm{~ms} \mathrm{LOW}$

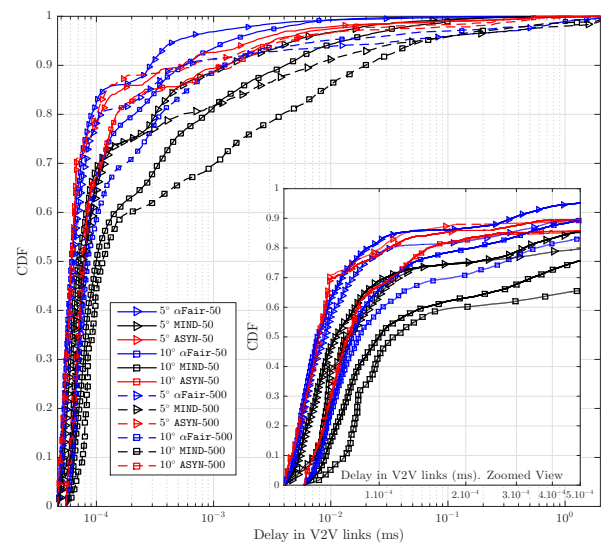

(d) Delay for $T_{s}=50 / 500 \mathrm{~ms} \mathrm{LOW}$

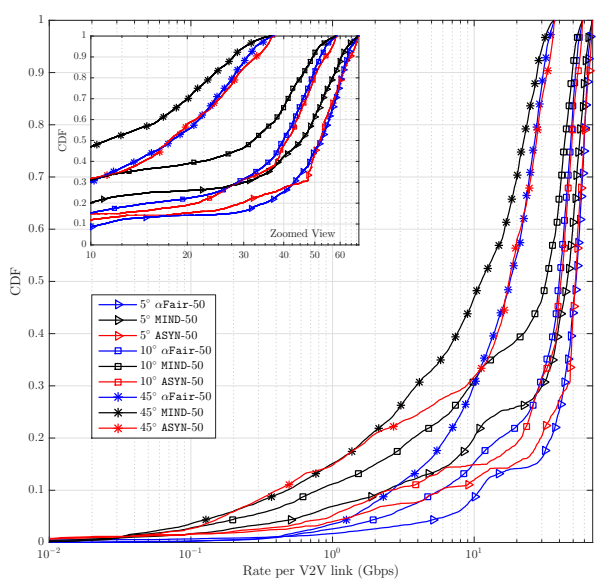

(b) Rate for $T_{s}=50 \mathrm{~ms} \mathrm{LOW}$

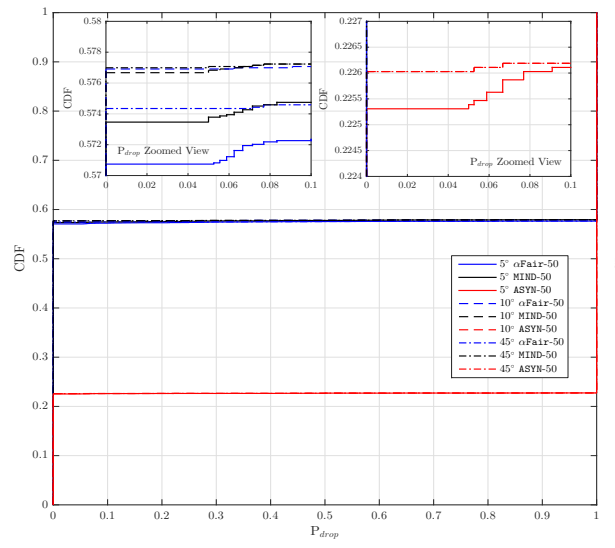

(e) Drops for $T_{s}=50 \mathrm{~ms} \mathrm{LOW}$

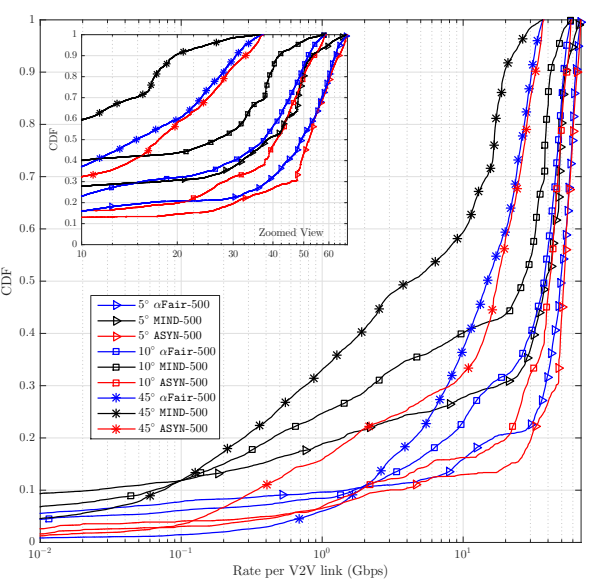

(c) Rate for $T_{s}=500 \mathrm{~ms} \mathrm{LOW}$

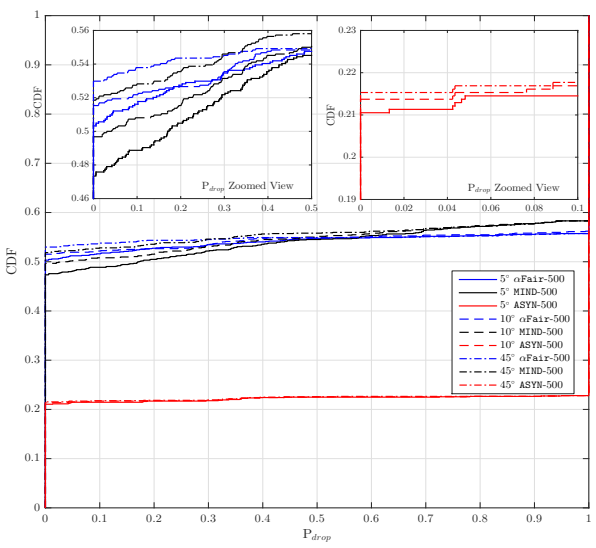

(f) Drops for $T_{s}=500 \mathrm{~ms} \mathrm{LOW}$

Fig. 2. Effect of scheduling interval selection on SINR (2a), rate (2b)-(2c), delay (2d), and drops (2e)-(2f) for LOW vehicle density, $\mathrm{P}_{s}=3200$ bits and traffic arrival rate of $1 / \lambda=1 / 2 \mathrm{~ms}$ scenario. Drop statistics $P_{\text {drop }}$ are obtained by aggregating transmission events over each scheduling period and queue, i.e. $\sum_{t=t_{s}}^{t_{s}+T_{t}}\left|\mathcal{A}_{i}^{\times}(t)\right| / \sum_{t=t_{s}}^{t_{s}+T_{t}} A_{i}(t) \forall t_{s}$. In (2a)-(2d) plots corresponding to $45^{\circ}$ have been omitted for the sake of clarity. Results in (2a)-(2f) have been computed from average V2 $\mathrm{V}$ pairing strategy success ratios of $95.09 \% \alpha \mathrm{Fair}-50,95.59 \% \mathrm{MIND}-50,35.79 \% \mathrm{ASYN}-50$ and ratios of $95.00 \% \alpha \mathrm{Fair}-500$, $96.72 \%$ MIND-500, 35.28\% ASYN-500, respectively.

The RRM methods considered are:

- $\alpha$-fair matching ( $\alpha$ Fair): based on the utilities learned from the previous scheduling interval as per (21) and (22) applies DA every $T_{s}$ ms to compute new V2V links.

- Minimum-distance baseline (MIND) where vTxs in the system trying to pair with their closest available vRx every $T_{s}$.

- Asynchronous long-term baseline (ASYN [9]): where every time a new vehicle enters the highway segment a pairing process is triggered. Specifically, two vehicles are paired if 1) they are eligible for pairing, i.e. still single and located within the first 20 meters of the highway segment; and 2) they are in the same or in adjacent lanes. V2V links will remain enforced until one of the vehicles in the link leaves the highway segment.

The maximum number of feasible V2V links in the highway segment at a given time slot will be subject to two natural limitations: the asymmetries in the number of current vTx and $\mathrm{vRx}$ and the coverage constraint that might yield unmatched
vTxs and vRxs due to an infeasible association between remaining available candidates. In spite of the former, the pairing strategy itself may impose additional restrictions. So, with the twofold aim of further evaluating the goodness of the RRM methods and of providing more context to simulation results, the ratio of the effectively associated vehicles under $\alpha$ Fair, and under both MIND and ASYN baselines will be quantified through the discussed cases.

Before proceeding with simulation results, we will briefly introduce the isolated effect of beamwidth selection, vehicle density, data traffic arrival rate together with packet size and of the scheduling interval length:

- Beamwidth selection: narrower beamwidths produce higher antenna gains as per (2). Those gains come, however, at the price of a longer beam alignment process that implies a reduced effective rate for those transmission slots where the alignment is accomplished as reflected by (4). In addition, for both very narrow and very wide beamwidths SINR will mainly depend on the strength of 


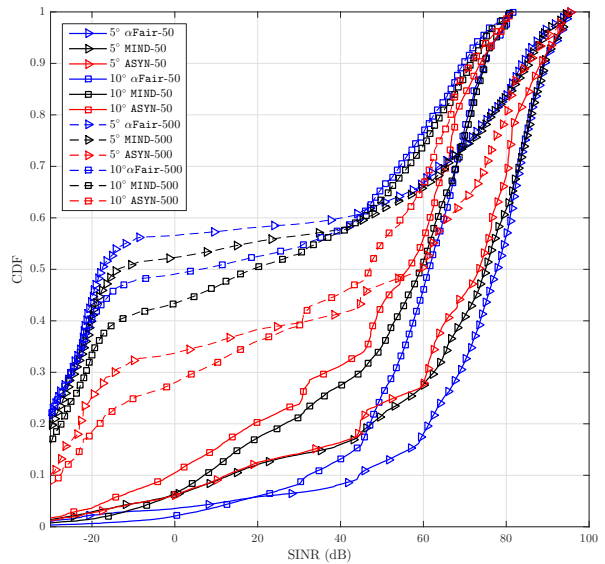

(a) SINR for $T_{s}=50 / 500 \mathrm{~ms}$ ULTRA

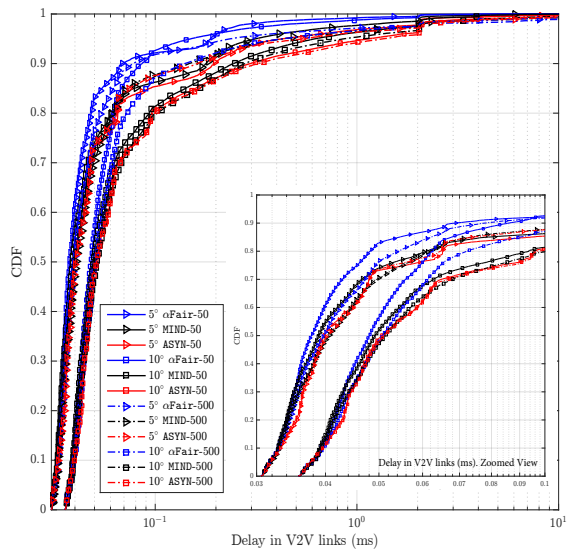

(d) Delay for $T_{s}=50 / 500 \mathrm{~ms}$ ULTRA

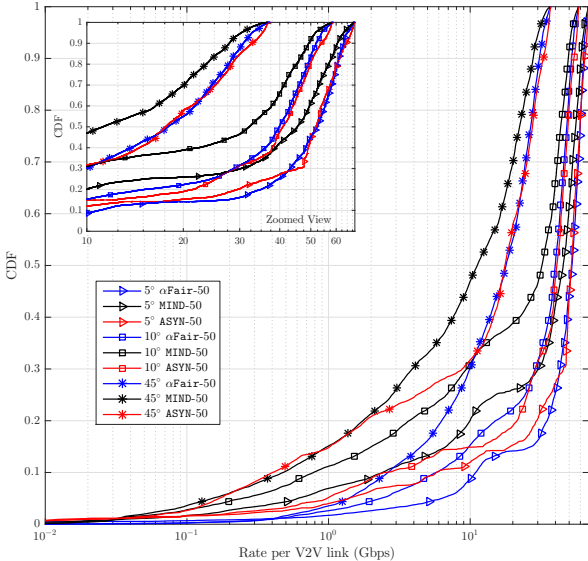

(b) Rate $T_{s}=50$ ULTRA

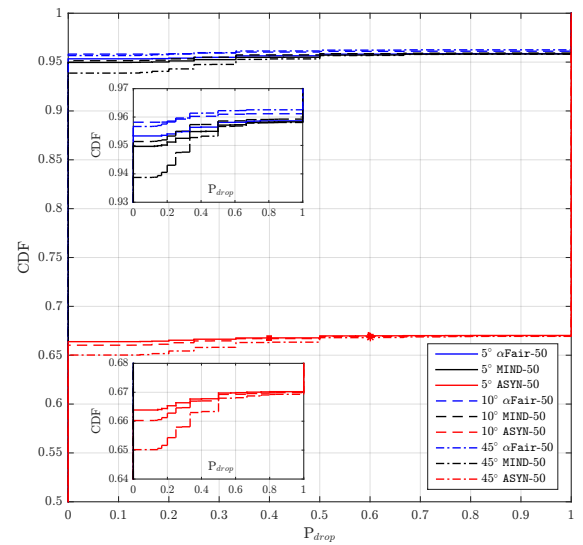

(e) Drops $T_{s}=50$ ULTRA

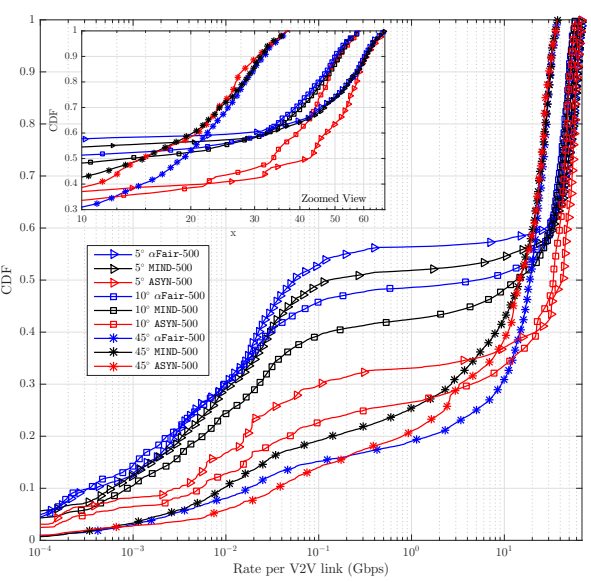

(c) Rate $T_{s}=500 \mathrm{~ms}$ ULTRA

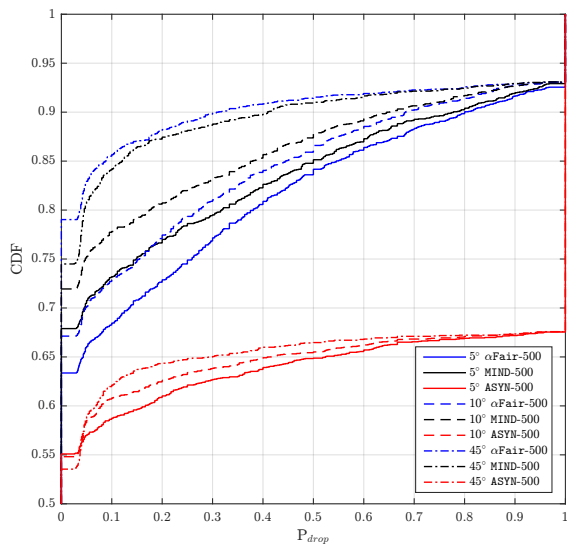

(f) Drops $T_{s}=500$ ULTRA

Fig. 3. Effect of scheduling interval selection on SINR (3a), rate (3b)-(3c), delay (3d) and drops (3e)-(3f) for ULTRA vehicle density, $\mathrm{P}_{s}=2097144$ bits and traffic arrival rate of $1 / \lambda=1 / 20 \mathrm{~ms}$ scenario. In (3a)-(3d) plots corresponding to $45^{\circ}$ have been omitted for the sake of clarity. Results in (3a)-(3f) have been computed from average V2V pairing strategy success ratios of 95.86\% $\alpha \mathrm{F}$ air-50, 95.94\% MIND-50, 67.34\% ASYN-50 and ratios of $96.01 \%$ $\alpha$ Fair-500, 95.99\% MIND-500, 67.08\% ASYN-500, respectively.

the desired link. Most of the interference will arrive to the sidelobes and to the mainlobe respectively and thus the effect of misalignment will be more severe.

- Vehicle density: disregarding the straightforward effect of vehicle density on the overall maximum number of simultaneous transmissions, higher vehicle densities increase the probability of having more blockers obtruding desired V2V links. In turn, with low vehicle density longer distances between vTx and vRx may result in worse channel conditions for active $\mathrm{V} 2 \mathrm{~V}$ links due to higher propagation losses, in vehicles being left unpaired for longer during the scheduling interval and leading to high drops if the vehicle who left the system was a vRx or in the overall number of feasible V2V links due to coverage constraint being reduced.

- Data traffic arrival rate and packet size: the first will effectively rule when a given vTx is activated for transmission whereas the second, in the absence of drops, will compel for how long it will remain active. So, at a given time slot, higher data traffic arrival rates and longer packet sizes will in general lead to more interference.

- Scheduling interval: shorter intervals will drive more frequent beam alignments that, on one hand, will reduce the likelihood of misalignment but, on the other, will increase the number of transmission slots affected by alignment delay. The significance of both opposed effects becoming more acute for narrower beamwidths.

\section{A. Discussion}

We will focus the discussion mainly on the two (vehicle density, $\mathrm{P}_{s}, 1 / \lambda$ ) combinations that produce use cases in opposite ends of the range: (LOW, 3200 bits, $1 / 2 \mathrm{~ms}$ ), to characterize frequent (500 packets per second) short-length messages exchanged between a limited number of vehicles; and (ULTRA, 2097144 bits, 1/20ms), which corresponds to long packets arriving at a lower rate but to a much more dense vehicle environment. Simulation results for both use cases are correspondingly collected in Fig. 2 and Fig. 3. In the remaining of this subsection we will assess the performance 
of the benchmark RRM methods in both scenarios under scheduling intervals of 50 and $500 \mathrm{~ms}^{5}$.

Performance statistics for (LOW, 3200 bits, 1/2ms) in Fig. 2 show in general little impact of the scheduling interval selection. As a reference, SINR in Fig. 2a indicates that for $\varphi=5^{\circ}$ around $10 \%, 5 \%$ and $3.5 \%$ of the transmission slots under MIND, $\alpha \mathrm{F}$ air and ASYN, correspondingly, are in outage range $(-20 \mathrm{~dB}$ or less). It is noticeable that ASYN seems to be the least affected by the scheduling interval selection no matter the beamwidth considered. However, only around $22 \%$ of its queues did not incur drops along the different scheduling intervals, and its association success reached barely $35 \%-$ for values around 50-60\% of drop-free events and of $95 \%$ association success of its counterparts-. As for $\alpha \mathrm{Fair}$, and MIND, with the latter coming always behind in performance, the probability of reaching a certain SINR is roughly $10 \%$ lower for $T_{s}=500 \mathrm{~ms}$. The increased likelihood of misalignment of $\mathrm{V} 2 \mathrm{~V}$ links inflicted by longer scheduling intervals does not greatly degrade the performance which seems to be hampered by the topology itself.

A detailed look into Fig. 3a and Figs. 3b-3c shows an altogether different case for (ULTRA, 2097144 bits, 1/20 ms): narrowest beamwidths provide the worst performance for $\mathrm{T}_{s}=500 \mathrm{~ms}$ in all three $\mathrm{V} 2 \mathrm{~V}$ association schemes with an upsurge on outage events from levels below $5 \%$ to over $40 \%$ for $5^{\circ}$ and $10^{\circ}$ beamwidths in $\alpha \mathrm{F}$ air and MIND. Moreover, a sharp downfall from $95 \%$ drop-free events from Fig. 3e to $63 \%$ and $66 \%$ in $\alpha \mathrm{F}$ air and MIND respectively is observed in Fig. 3f.

Comparing Figs. 2d-3d and Figs. 2e-3e we can observe that increasing $P_{s}$ and, at the same time, reducing $1 / \lambda$ yields, as expected, longer delays, but also far less drops -specially with $T_{s}=50 \mathrm{~ms}-;$ In fact, complementary simulation, omitted due to space limitations, were conducted for both LOW and ULTRA scenarios and $\mathrm{P}_{s}=[3200,2097144]$ bits with $1 / \lambda=\left[1 / T_{t}\right.$, $\left.1 / 3 T_{t}, 1 / 10 T_{t}, 1 / 30 T_{t}\right] \mathrm{ms}$ and confirmed that the system is, in terms of reliability, much more sensitive to variations on the traffic inter-arrival rate than to packet size. Also noteworthy is that $\alpha \mathrm{F}$ air, which relies in learning techniques and in general outperforms all other approaches, produces the worst results for $T_{s}=500 \mathrm{~ms}$, which suggests that the weighted average learning over such a long period is not efficient, not even in a rectilinear vehicular scenario as the one analyzed in this work.

\section{Conclusions And Future Research Directions}

This paper has elaborated on the interplay among the scheduling period, the data traffic arrival process and the density of vehicles in a straight line highway segment for a benchmark of three V2V link allocation RRM strategies. Simulation results highlight that resorting to narrowest beamwidths, even in a simplistic vehicle movement simulation that considers

\footnotetext{
${ }^{5}$ Notice here that delay and reliability $-\mathrm{P}_{d r o p}-$ statistics should be jointly considered for a proper interpretation of the results; the queue dropping policy leads to filtering out from delay calculation packets not fulfilling a delay below $D_{\max }^{\lambda}$ set by the traffic arrival rate $1 / \lambda$. Consequently, only packets on fast enough flushing queues contribute to delay figures.
}

constant speeds and no deviation in the x-axis from the lane center, might not produce the best performance should the scheduling period and, thus, the beam realignment frequency not be carefully selected so as to suit actual vehicle density and data traffic conditions. The good performance of our matching framework over a comprehensive number of configurations has been also validated. Future research will be directed towards assessing the performance in non-linear road networks subject to very frequent misalignments between vehicles, for which we expect that the scheduling period/beamwidth selection will play a decisive role and where, most likely, a certain level of beam-tracking/beam realignment will be essential.

\section{ACKNOWLEDGMENTS}

This work was supported by Basque Government ELKARTEK program BID3ABI project and by the Spanish Ministerio de Economia y Competitividad (MINECO) under grant TEC2016-80090-C2-2-R (5RANVIR).

\section{REFERENCES}

[1] T. S. Rappaport, S. Sun, R. Mayzus, H. Zhao, Y. Azar, K. Wang, G. N Wong, J. K. Schulz, M. Samimi, and F. Gutierrez, "Millimeter wave mobile communications for 5g cellular: It will work!" IEEE Access, vol. 1, pp. 335-349, 2013.

[2] J. G. Andrews, S. Buzzi, W. Choi, S. V. Hanly, A. Lozano, A. C. K. Soong, and J. C. Zhang, "What Will 5G Be?" IEEE J. Sel. Areas Commun., vol. 32, no. 6, pp. 1065-1082, jun 2014.

[3] "5G-PPP White Paper on Automotive Vertical Sector," 5G-PPP, Tech. Rep., 2015. [Online]. Available: https://5g-ppp.eu/white-papers/

[4] N. Lu, N. Cheng, N. Zhang, X. Shen, and J. W. Mark, "Connected vehicles: Solutions and challenges,' IEEE Internet Things Journal, vol. 1, no. 4, pp. 289-299, aug 2014.

[5] J. B. Kenney, "Dedicated short-range communications (DSRC) standards in the United States," Proc. IEEE, vol. 99, no. 7, pp. 1162-1182, 2011.

[6] J. Choi, N. Gonzalez-Prelcic, R. Daniels, C. R. Bhat, and R. W. Heath Jr, "Millimeter wave vehicular communication to support massive automotive sensing," arXiv preprint arXiv:1602.06456, 2016.

[7] P. Kumari, N. Gonzalez-Prelcic, and R. W. Heath, "Investigating the ieee 802.11 ad standard for millimeter wave automotive radar," in Proc. IEEE Veh. Technol. Conf. Fall (VTC), 2015, pp. 1-5.

[8] V. Va, T. Shimizu, G. Bansal, and R. W. Heath, "Beam design for beam switching based millimeter wave vehicle-to-infrastructure communications," in Proc. IEEE Int. Conf. Commun. (ICC), 2016, pp. 1-6.

[9] —_ "Millimeter Wave Vehicular Communications: A Survey," Foundations and Trends in Networking, p. 107, 2016.

[10] C. Perfecto, J. Del Ser, and M. Bennis, "Millimeter Wave V2V Communications: Distributed Association and Beam Alignment," arXiv preprint arXiv:1612.04217, 2016.

[11] A. Yamamoto, K. Ogawa, T. Horimatsu, A. Kato, and M. Fujise, "Pathloss prediction models for intervehicle communication at $60 \mathrm{ghz}$," IEEE Trans. Veh. Technol., vol. 57, no. 1, pp. 65-78, 2008.

[12] J. Wang, Z. Lan, C.-W. Pyu, T. Baykas, C.-S. Sum, M. Rahman, J. Gao, R. Funada, F. Kojima, H. Harada, and S. Kato, "Beam codebook based beamforming protocol for multi-gbps millimeter-wave wpan systems," IEEE J. Sel. Areas Commun., vol. 27, no. 8, pp. 1390-1399, 2009.

[13] H. Shokri-Ghadikolaei, L. Gkatzikis, and C. Fischione, "Beam-searching and transmission scheduling in millimeter wave communications," in Proc. IEEE Int. Conf. Commun. (ICC), 2015, pp. 1292-1297.

[14] Y. Gu, W. Saad, M. Bennis, M. Debbah, and Z. Han, "Matching theory for future wireless networks: fundamentals and applications," IEEE Commun. Mag., vol. 53, no. 5, pp. 52-59, 2015.

[15] D. Gale and L. S. Shapley, "College admissions and the stability of marriage," The American Mathematical Monthly, vol. 69, no. 1, pp. 9$15,1962$.

[16] J. Mo and J. Walrand, "Fair end-to-end window-based congestion control," IEEE/ACM Trans. Netw., vol. 8, no. 5, pp. 556-567, 2000. 